\title{
Assessment of Right Ventricular Function by Newer Imaging in Echocardiography in Idiopathic Pulmonary Arterial Hypertension
}

\author{
Priyanker Mondal ${ }^{\mathrm{a}}$, Prashant Kumar ${ }^{\mathrm{a}, \mathrm{c}}$, Manish Vinayak ${ }^{\mathrm{b}}$, Anurag Passi ${ }^{\mathrm{a}}$, \\ Dhurjati Prasad Sinha ${ }^{\mathrm{a}}$
}

\begin{abstract}
Background: The aims of the study were to assess the right ventricular (RV) functions in patients with idiopathic pulmonary arterial hypertension (IPAH) with RV longitudinal strain (RVLS) in addition to conventional parameters, as well as its correlation with severity and prognosis in IPAH.
\end{abstract}

Methods: Twenty-two IPAH patients were followed up for 1 year. ANOVA and Gabriel's pairwise comparison tests were used for comparison of RVLS with respect to WHO functional class status. Patients were divided into non-survival (group 1) and survival (group 2), and clinical and echocardiographic parameters of RV function were compared at baseline and at 6 months with $t$-test $\&$ Mann-Whitney test.

Results: At baseline, with respect to WHO functional class, mean RVLS showed no significant interclass difference $(\mathrm{P}=0.0781)$. Among the other conventional echocardiographic parameters, RV E/A showed significant difference at baseline $(\mathrm{P}=0.004)$, but not at 6 months $(\mathrm{P}=0.366)$; whereas tricuspid annular plane systolic excursion (TAPSE) which had no significant difference initially $(\mathrm{P}=0.174)$ revealed a significance level at 6 months $(\mathrm{P}=0.029)$ between the two groups. Fractional area change (FAC), RV index of myocardial performance (RIMP), and right atrial (RA) area displayed significant difference neither at baseline nor at 6 months. RVLS exhibited significant difference neither at baseline $(\mathrm{P}=0.912)$ nor at 6 months $(\mathrm{P}=0.181)$. None of the echocardiographic parameters including RVLS showed a significant average change with change in severity of PAH both at 6 and 12 months.

Conclusion: RVLS was not proved to be a useful parameter for early detection of RV dysfunction and prognosis in patients with IPAH in comparison with the conventional echocardiographic parameters.

Keywords: RV longitudinal strain; Idiopathic pulmonary arterial hy-

Manuscript submitted August 26, 2017, accepted September 13, 2017

${ }^{a}$ Department of Cardiology, I.P.G.M.E \& R and S.S.K.M Hospital, Kolkata, India

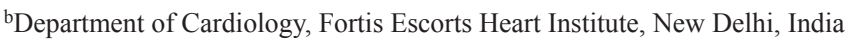
${ }^{c}$ Corresponding Author: Prashant Kumar, Department of Cardiology, I.P.G.M.E \& R and S.S.K.M Hospital, Kolkata, India.

Email: pkumar_rims@yahoo.com

doi: https://doi.org/10.14740/cr600w pertension; RV dysfunction

\section{Introduction}

Idiopathic pulmonary arterial hypertension (IPAH), a rare and progressive disease with a poor prognosis, is caused by obliterative pulmonary vasculopathy of yet unknown etiology. The ability of the right ventricle to adapt to the progressive increase in pulmonary vascular resistance (PVR) associated with changes to the pulmonary vasculature in PAH is the main determinant of a patient's functional capacity and survival [1].

The World Health Organization functional class (WHOFC) remains one of the most powerful predictors of survival, not only at diagnosis, but also during follow-up [2, 3]. Apart from exercise pulmonary arterial systolic pressure (PASP), PVR and right atrial (RA) pressure, none of the conventional echocardiographic parameters of RV function proved to be a good indicator of prognosis. RA pressure, cardiac index (CI) and mixed venous oxygen saturation $\left(\mathrm{MvO}_{2}\right)$ measured during right heart catheterization (RHC) are the most robust indicators of RV function and prognosis, whereas mean pulmonary arterial pressure (mPAP) provides little prognostic information (except for calcium channel blocker responders) [2, 3]. Serial assessment of right ventricular longitudinal strain (RVLS) in patients of PAH had been shown to be an independent predictor of clinical deterioration and mortality [4]. Few studies also showed the usefulness of RVLS in children with IPAH [5].

The objective of our study was to determine whether RVLS could be used as an important predictor of prognosis in IPAH or not, in Indian population irrespective of PAH-specific therapy.

\section{Methods}

\section{Patient selection}

We conducted an institutional-based, single-centered, prospective study from March 2015 to August 2016. Patients were included if they had met following diagnostic criteria: resting $\mathrm{mPAP}>25 \mathrm{~mm} \mathrm{Hg}$ with pulmonary capillary wedge pressure (PCWP) $<15 \mathrm{~mm} \mathrm{Hg}$ and PVR $>3$ Wood Units measured 


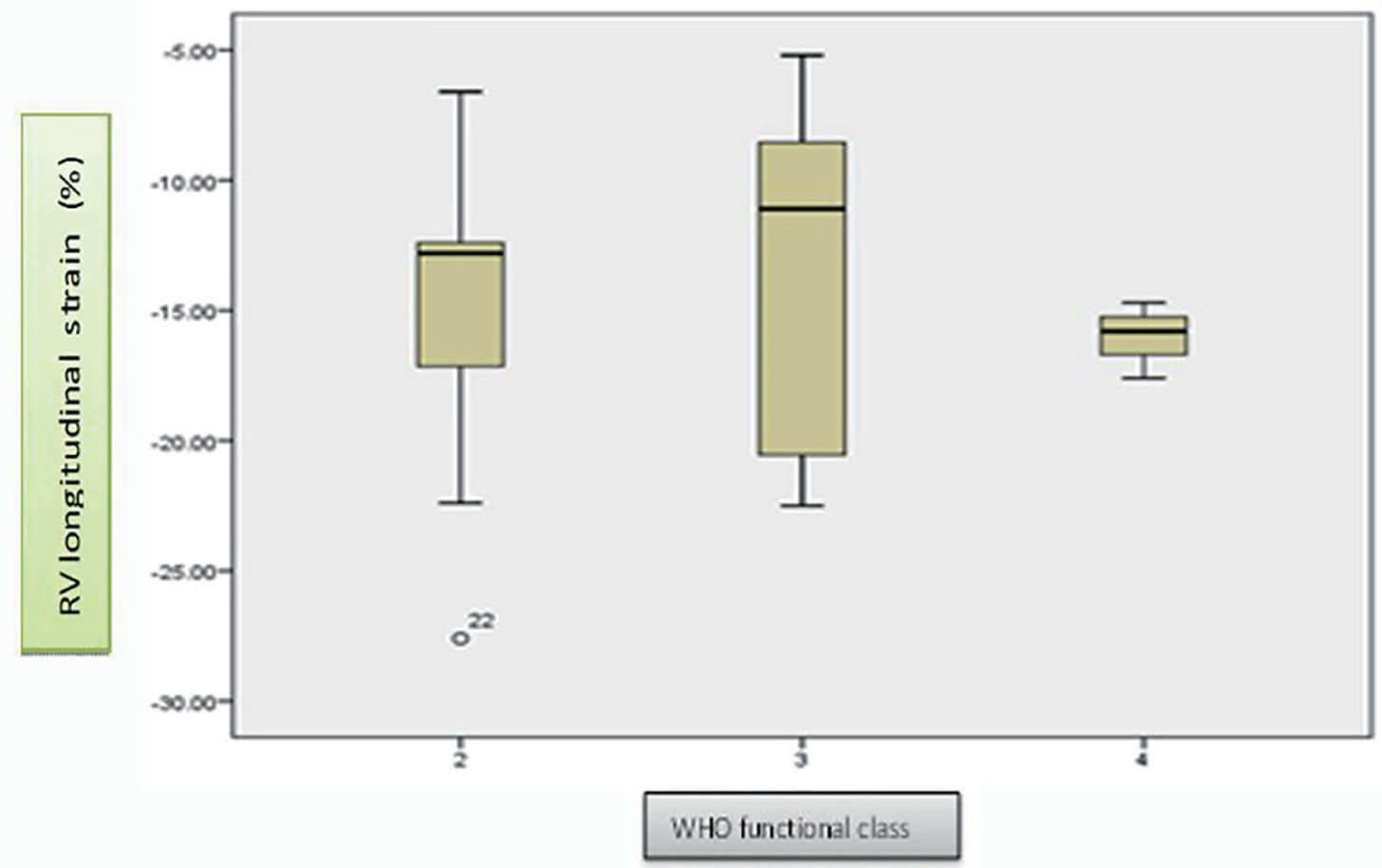

Figure 1. Whisker plot diagrams showing distribution of RV longitudinal strain in different WHO functional class at baseline. At baseline, there was no significant interclass difference in mean RV longitudinal strain distribution $(P=0.0781)$.

by cardiac catheterization apart from the range of additional investigations to exclude other secondary causes.

\section{Study protocol}

Physical examination, laboratory investigation, detailed echocardiographic evaluation, right heart catheterization, 6-min walking distance (6MWD), and peripheral capillary oxygen saturation $\left(\mathrm{SpO}_{2}\right)$ measurement were undertaken before starting PAH-specific treatment at baseline and 6 monthly thereafter according to functional status. Following echocardiographic parameters were measured: 1) RV diastolic parameters - RV E/A; 2) RV systolic parameters including tricuspid annular plane systolic excursion (TAPSE), lateral annular S, fractional area change (FAC), RV index of myocardial performance (RIMP), and RVLS; 3) RA area and mean pressure; 4) PASP and mPAP; 5) PVR; 6) RV basal, mid cavity, right ventricular outflow tract (RVOT) and longitudinal diameters, and subcostal thickness; and 7) eccentricity index. Patients were divided into non-survival (group 1) and survival (group 2), and clinical and echocardiographic parameters of RV function were compared at baseline and at 6 and 12 months. The study protocol was approved by the Institutional Ethics Committee of our hospital.

\section{Treatments}

All patients were put on phosphodiesterase- 5 inhibitor and/or endothelin receptor antagonist. Only one of the patients was vaso-reactive who received high-dose calcium channel blocker (CCB). WHO class IV patients received intravenous epoprostenol infusion.

\section{Statistical analysis}

Twenty-two patients of IPAH were followed up from March 2015 to August 2016. ANOVA and Gabriel's pairwise comparison tests were used for comparison of RVLS with respect to WHO functional class status. Patients were divided into non-survival (group 1) and survival (group 2), and clinical and echocardiographic parameters of RV function were compared at baseline and 6 and 12 months (wherever possible) with $t$-test and Mann-Whitney test depending upon pattern of distribution. Analysis was also done to evaluate the level of change the individual parameters were showing in respect to change in severity status over time by Kruskal-Wallis test.

\section{Results}

\section{Baseline characteristics}

There were 15 female (68\%) and seven male (32\%) patients in our study. Five patients ( $22.7 \%$ of study population) died during follow-up period, out of which three $(60 \%)$ were female. The mean ages of survivors and non-survivors were $30.70 \pm$ 


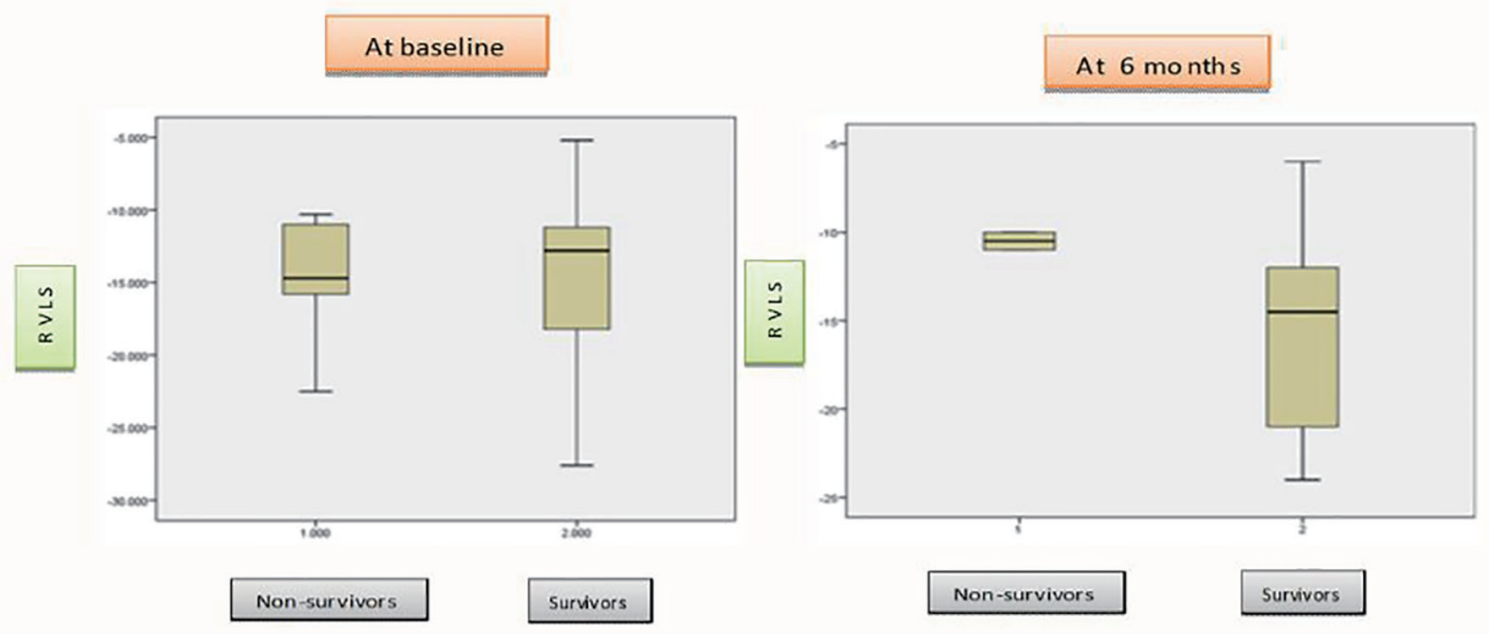

Figure 2. Whisker plot diagrams showing average differences of RV longitudinal strain between non-survivors and survivors groups at baseline and at 6 months. The difference in average RV longitudinal strain between non-survivors and survivors groups neither significant at baseline $(P=0.912)$ nor at 6 months $(P=0.181)$.

18.97 and $31.10 \pm 16.54$ years, respectively, with a range of 3 - 67 years at the diagnosis. The difference in mean age was not significant between the two groups $(\mathrm{P}=0.967)$. At the time of diagnosis, 11 patients were in WHO functional class II, eight in class III, and three in class IV. At baseline, with respect to WHO functional class, mean RVLS showed no significant interclass difference $(\mathrm{P}=0.0781)$ (Fig. 1).

\section{Follow-up data}

When $\mathrm{SpO}_{2}$ and 6MWD were compared between the two groups (survival and non-survival), the difference was significant both at baseline $(\mathrm{P}=0.002$ and $\mathrm{P}=0.009$, respectively) and at 6 months $(\mathrm{P}=0.020$ and $\mathrm{P}=0.035$, respectively). Among the other conventional echocardiographic parameters, RV E/A showed significant difference at baseline $(P=0.004)$, but not at 6 months $(\mathrm{P}=0.366)$; whereas TAPSE which had no significant difference initially $(\mathrm{P}=0.174)$ revealed a significance level at 6 months $(\mathrm{P}=0.029)$ between the two groups. FAC, RIMP, and RA area displayed significant difference neither at baseline nor at 6 months. RVLS exhibited significant difference neither at baseline $(\mathrm{P}=0.912)$ nor at 6 months $(\mathrm{P}=$ 0.181) (Fig. 2). Analysis was also done by Kruskal-Wallis test to check whether average change in grade of severity of PAH associated with significant average change in any parameters over time or not. None of the echocardiographic parameters including RVLS showed a significant average change with change in severity of PAH both at 6 and 12 months (Tables 1 and 2).

\section{Discussion}

This was the first study to assess serial RV function and its relation to prognosis using 2D strain echocardiography in addi- tion to conventional parameters in India. Of the study subjects, $68 \%$ were female. This female preponderance was in conformity with the NIH and the current day REVEAL registries where F/M were 2:1 and 4:1, respectively. Mortality was noted in $22.7 \%$ of the study subjects which was comparable to French registry (29\%) [6], but much lower than NIH (55\%) [7] and a study by Nickel et al (49\%) [3]. Though the difference in mean age was not significant between the two groups in our study, the age at diagnosis ranged from 3 to 67 years. Even though the mean age at diagnosis was 37 in the NIH registry and approximately 50 in the more recent registries, IPAH can affect children and adults into their 70s [7]. Conventional echocardiographic parameters, such as RV fractional area change, mPAP, PASP, RIMP, TAPSE, and tricuspid annular peak systolic velocity, had been used earlier as follow-up tool for severity assessment and prognosis in adults with PAH. Newer 2D speckle tracking strain imaging assessing percentage change in myocardial deformation had been used primarily to assess left ventricular function, but more recently, it had also been applied in the assessment of RV regional and global function $[8,9]$.

The 6MWD, $\mathrm{SpO}_{2}$, WHO-FC, exercise PASP, catheterization-derived $\mathrm{CI}$, mean RA pressure, and $\mathrm{MvO}_{2}$ are powerful indicators of severity and prognosis in PAH. Hardegree et al [4] showed significant improvement of mean RVLS after $\mathrm{PAH}$-specific treatment initiation in patients with group $1 \mathrm{PAH}$ of comprehensive clinical classification of pulmonary hypertension and after adjusting for age, functional class, and RV strain at baseline, patients with $\geq 5 \%$ improvement in RV free wall systolic strain had a greater than sevenfold lower mortality risk at 4 years (hazard ratio: $0.13,95 \%$ confidence interval: $0.03-0.50, \mathrm{P}=0.003$ ). In our study, with respect to WHO-FC at baseline, mean RVLS showed no significant interclass difference $(P=0.0781)$. RVLS was not able to detect any difference between survival and non-survival group. No significant change was observed in RVLS over time during follow-up 
Table 1. Distribution of Average Change in Different Parameters Between Baseline and 6 Months With Respect to Change in Severity of PAH

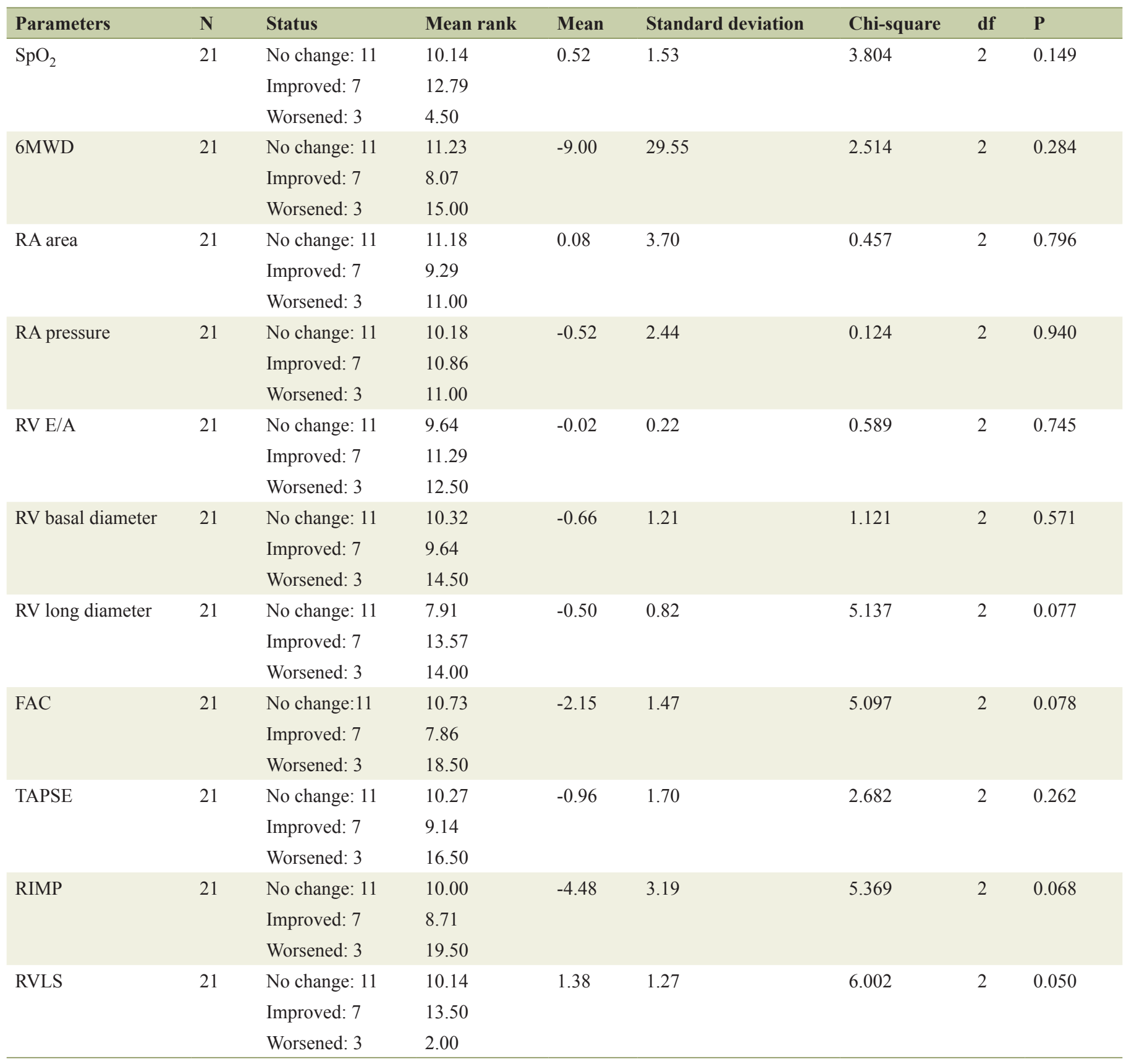

The average change in no parameter achieved significance level with change in severity of PAH at 6 months with comparison to baseline. Out of 22 patients, one is yet to come for follow-up at 6 months.

with changes in severity status on treatment. The variability of measurement of RVLS which was angle-dependent might have affected the result besides inadequate sample size.

\section{Limitations}

This was a small, retrospective, single-center study with in- herent limitations. Age-matched controls were not taken. So, the analysis derived data do not reflect changes or significance in comparison to normal population. Moreover, we did not measure strain rate as it could not be measured with our echomachine GE VIVID S6. Strain rate might better reflect contractility than strain and might be less influenced by loading conditions. Some were receiving pulmonary vasodilator even before enrolment in this study. These factors might have af- 
Table 2. Distribution of Average Change in Different Parameters Between 6 and 12 Months With Respect to Change in Severity of $\mathrm{PAH}$

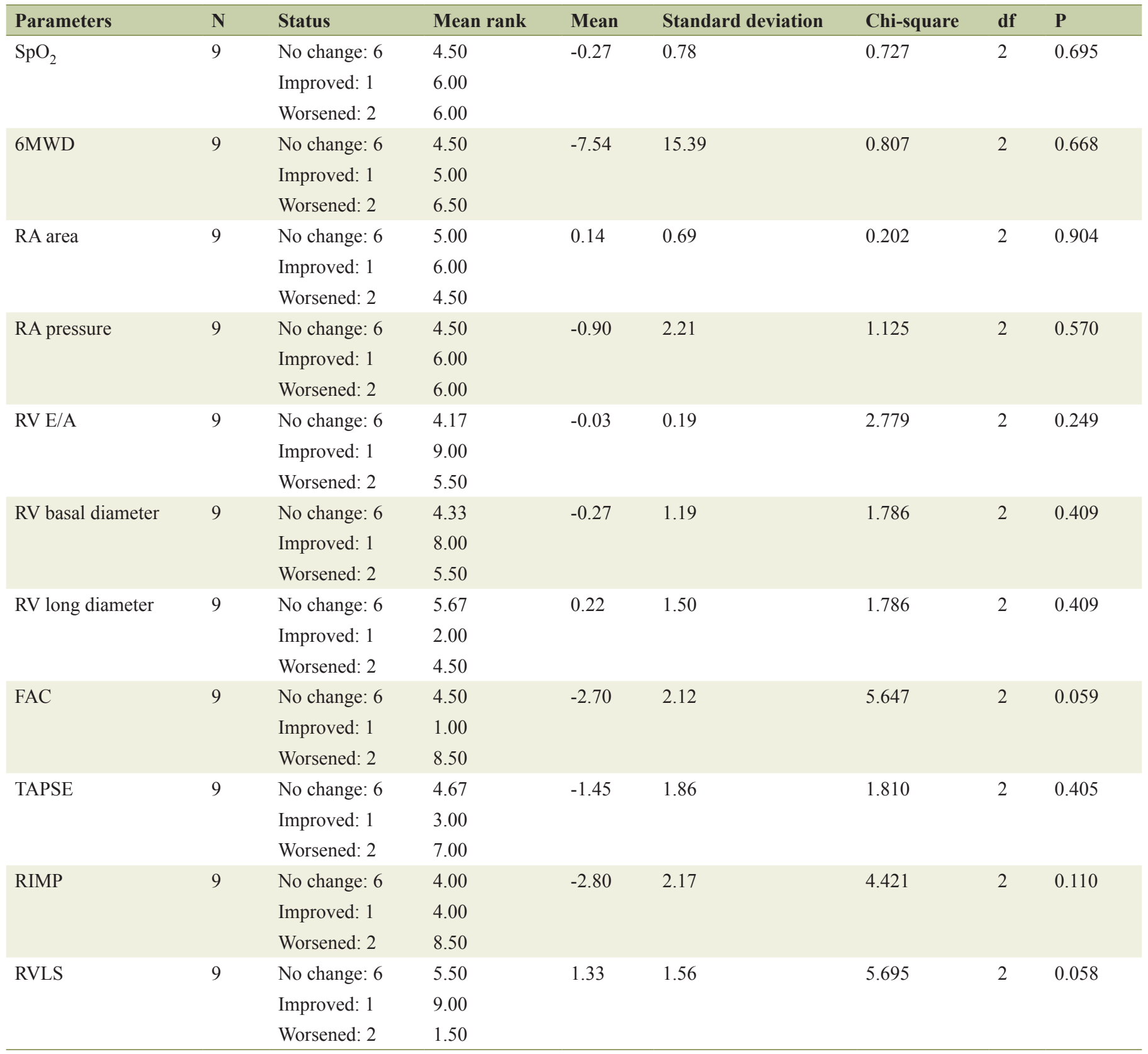

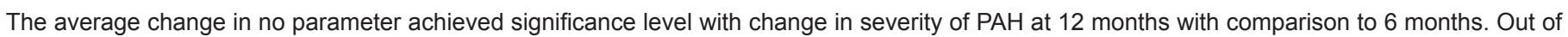
21 patients, nine patients yet to reach their 12 months follow-up and three patients expired at 6 months follow-up.

fected the result.

\section{Conclusion}

The present study revealed the RV longitudinal strain was not proved to be a useful parameter for early detection of RV dysfunction in patients with IPAH in comparison with the conventional echocardiographic parameters. Moreo- ver, it failed to qualify as a tool to judge the prognosis of the same. Further large multicenter studies are needed to evaluate the role of the RV longitudinal strain in Indian population.

\section{Competing Interests}

None. 


\section{Grant Support}

None.

\section{Financial Disclosure}

None.

\section{References}

1. Kovacs G, Berghold A, Scheidl S, Olschewski H. Pulmonary arterial pressure during rest and exercise in healthy subjects: a systematic review. Eur Respir J. 2009;34(4):888-894.

2. Sitbon O, Humbert M, Nunes H, Parent F, Garcia G, Herve $\mathrm{P}$, Rainisio $\mathrm{M}$, et al. Long-term intravenous epoprostenol infusion in primary pulmonary hypertension: prognostic factors and survival. J Am Coll Cardiol. 2002;40(4):780788.

3. Nickel N, Golpon H, Greer M, Knudsen L, Olsson K, Westerkamp V, Welte T, et al. The prognostic impact of follow-up assessments in patients with idiopathic pulmonary arterial hypertension. Eur Respir J. 2012;39(3):589596.

4. Hardegree EL, Sachdev A, Villarraga HR, Frantz RP, McGoon MD, Kushwaha SS, Hsiao JF, et al. Role of serial quantitative assessment of right ventricular function by strain in pulmonary arterial hypertension. Am J Cardiol. 2013;111(1):143-148.

5. Okumura K, Humpl T, Dragulescu A, Mertens L, Friedberg MK. Longitudinal assessment of right ventricular myocardial strain in relation to transplant-free survival in children with idiopathic pulmonary hypertension. J Am Soc Echocardiogr. 2014;27(12):1344-1351.

6. Humbert M, Sitbon O, Chaouat A, Bertocchi M, Habib G, Gressin V, Yaici A, et al. Pulmonary arterial hypertension in France: results from a national registry. Am J Respir Crit Care Med. 2006;173(9):1023-1030.

7. D'Alonzo GE, Barst RJ, Ayres SM, Bergofsky EH, Brundage BH, Detre KM, Fishman AP, et al. Survival in patients with primary pulmonary hypertension. Results from a national prospective registry. Ann Intern Med. 1991;115(5):343-349.

8. Borges AC, Knebel F, Eddicks S, Panda A, Schattke S, Witt C, Baumann G. Right ventricular function assessed by two-dimensional strain and tissue Doppler echocardiography in patients with pulmonary arterial hypertension and effect of vasodilator therapy. Am J Cardiol. 2006;98(4):530-534.

9. Fukuda Y, Tanaka H, Sugiyama D, Ryo K, Onishi T, Fukuya H, Nogami M, et al. Utility of right ventricular free wall speckle-tracking strain for evaluation of right ventricular performance in patients with pulmonary hypertension. J Am Soc Echocardiogr. 2011;24(10):11011108. 